\title{
Medical Evaluation for Driver Qualification for Patients With Cardiovascular Disorders
}

\author{
Ram Kakaiya, $M D$, and Pbillip Fulkerson, $M D$
}

Background: Primary care physicians commonly care for patients whose disabilities caused by cardiovascular diseases result in difficulty driving an automobile. Patients often seek their physician's advice as to whether they can continue to drive safely.

Metbods: A MEDLINE literature search was performed from 1966 to 2000, using the query terms "automobile driving" and "automobile driving and cardiac." Selected articles were reviewed, as well as additional articles found through review of the references. In some cases articles were found by searching the Internet using the above key words and new query terms guided by the results of the original search.

Results and Conclusions: The most important factor determining eligibility is whether the patient reports manifestations of cerebral hypoxia. Laboratory procedures are available for patients in whom the decision is difficult. Sometimes a referral to a specialist is needed. Many patients with a known cardiovascular disorder can continue to drive safely. Some conditions require a waiting period before driving can be resumed, while others call for a complete cessation of driving. Several guidelines from the literature are listed that can be helpful to primary care physicians when dealing with questions from their patients on this important topic. (J Am Board Fam Pract 2000;13:261-7.)

Primary care physicians commonly care for patients who have cardiovascular diseases. Disabilities caused by these illnesses can result in difficulty driving an automobile. As a result, patients often seek their physician's advice as to whether they can continue to drive safely. To provide this guidance, primary care physicians should be familiar with the current recommendations for patients with cardiovascular diseases. We provide a summary of these recommendations.

\section{Methods}

A MEDLINE literature search was performed from 1966 to 2000 , using the query terms "automobile driving" and "automobile driving and cardiac." The abstracts were reviewed to select articles of interest. Selected complete articles were retrieved and reviewed. Additional citations of interest were selected from the references listed in these

Submitted, revised, 24 January 2000.

From the Primary Care Clinic at Rockton (RK, PF), University of Illinois College of Medicine at Rockford, Rockton, Ill. Address reprint requests to Ram Kakaiya, MD, University of Illinois College of Medicine at Rockford, Primary Care Clinic at Rockton, 1511 Blackhawk Boulevard, Rockton, IL 61072. papers and were also retrieved and reviewed. In some cases, articles were obtained on the Internet by using similar query terms, with additional searching performed by new query terms guided by the results of the original search.

\section{Motor Vehicle Driver License Regulations}

In the United States, issuance of a private vehicle driver license is regulated by each state, independently. Federal laws govern the issuance of a driver's license to operate a commercial motor vehicle in interstate commerce. ${ }^{1}$ The federal laws are applied uniformly throughout the United States, and they can provide useful guidance on a number of medical conditions including the cardiovascular diseases. Likewise, state laws on this topic have begun to evolve. By 1991, according to Drickamer and Marottoli, ${ }^{2}$ approximately one half of the states had already implemented regulations to limit driving by patients who have had an episodic loss of consciousness. One fifth of the states also had laws regarding patients with cardiac arrhythmia. ${ }^{2}$ Cardiovascular diseases are the second leading cause of driver limitation issues referred to the departments of motor vehicles of the 37 states reported by Drickamer and Marottoli, exceeded only by sei- 
zures. $^{2}$ In addition, most jurisdictions already have strict guidelines for addressing driving privileges for persons who have seizure disorders.

Increasing regulatory attention at the state level is understandable, because deaths caused by coronary ischemic heart disease are among the most common natural causes of death. In addition, sudden driver incapacitation could cause a collision, injuring passengers and innocent bystanders as well as the driver. In two studies on death while driving, coronary disease was responsible for $44 \%$ to $88 \%$ of driver deaths from natural causes. These fatalities do not include those resulting from trauma or alcohol. ${ }^{3,4}$ When all automobile-related deaths are considered, not just the known sudden natural deaths at the wheel, data reported by Halinen and Jaussi $^{5}$ show that a probable cardiac arrest was the cause of about $2 \%$ of all drivers' deaths. Thus, it is clearly important to recognize and avoid cardiac disease as a cause of sudden death while driving.

\section{Medical History and Physical Examination}

Among the most important benchmarks influencing a decision about continuation of driving is a patient complaint suggesting cerebral ischemia. Federal laws generally disqualify persons with cardiovascular disease for a commercial motor vehicle license if they have symptoms of syncope, dyspnea, collapse, or congestive heart failure. ${ }^{1}$ The medical history can also give clues about the risk for sudden death, such as symptoms consistent with untreated severe disease of the left main coronary artery (unstable, crescendo, or incapacitating angina) or sustained ventricular tachycardia on a Holter monitor. It is also helpful to know whether these cardiac symptoms are chronic, acute, or paroxysmal. For example, an isolated case of syncope from a vasovagal attack is not a contraindication to driving, but recurrent altered states of consciousness caused by chronic orthostatic hypotension should be investigated, as they might be sufficiently disabling to contraindicate driving. The physician who has thorough knowledge of each patient's medications will be aware of the possibility of adverse systemic or cardiac effects. $\beta$-Blockers, in particular, can cause weakness and orthostatic hypotension, compromising safe driving.

Findings from a physical examination are less helpful, although the examination can detect profound skeletal muscle weakness associated with a low cardiac output in severe cases of chronic congestive heart failure. An $\mathrm{S}_{3}$ gallop or pulsus alternans also suggests seriously impaired ventricular performance. Cardiac murmurs generally do not contraindicate driving, with the exception of those associated with valvular and hypertrophic subvalvular stenosis; both have a propensity to syncope and sudden death. Cyanosis suggests reduced oxygen delivery to the peripheral circulation and impending cerebral ischemia. An irregular or rapid pulse might indicate a serious cardiac arrhythmia. Confusion or delirium, which can be detected readily during the physical examination, is an obvious contraindication to safe driving. Guidelines as they relate to cardiac symptoms and signs are displayed in Table $1 .^{6-8}$

\section{Laboratory Studies}

Cardiac patients can be evaluated by laboratory studies. These investigations can include a functional assessment by exercise testing, measurement of left ventricular ejection fraction and valvular function by echocardiography, and detection and assessment of severity of cardiac arrhythmia using a Holter monitor. Kavanagh et $\mathrm{al}^{9}$ reported that engaging in driving can cause physiologic cardiovascular changes. They detected an increase in peak heart rate to 101 beats per minute. Such changes can be assessed by having the patient wear a Holter monitor during the driving task. For patients who are known to have coronary artery disease, coronary angiography, a stress-radionuclide myocardial perfusion scan, or stress-echocardiography can be helpful. Negative findings with one or more of these tests are sufficient to recommend continuation of driving.

Physical working capacity during exercise testing is measured using the metabolic equivalent (MET), which is a valuable index of functional capacity of the patient. ${ }^{6}$ METs can be used to assign patients to certain levels of physical working capacity: class I ( 7 or more METs), class II ( 5 to 7 METs), class III (2 to 4 METs) and class IV (less than 2 METs). ${ }^{6}$ This classification generally corresponds to the functional classification of the New York Heart Association. This latter classification can be readily determined in the clinic by a careful medical history. The patients with a class I to III status need additional evaluation to judge driving safety. ${ }^{6}$ Those with a class IV functional status with 
Table 1. Guidelines for Automobile Driving by Patients with Cardiac Symptoms and Signs.

\begin{tabular}{|c|c|c|c|c|c|c|}
\hline \multirow[b]{2}{*}{ Condition } & \multicolumn{3}{|c|}{ Private Vehicle Driving } & \multicolumn{3}{|c|}{ Commercial Vehicle Driving } \\
\hline & USA & England & Canada & USA & England & Canada \\
\hline $\begin{array}{l}\text { Syncope, dyspnea, collapse, or uncompensated } \\
\text { heart failure }\end{array}$ & 2 & 2 & 2 & 2 & 2 & 2 \\
\hline $\begin{array}{l}\text { Confusion, delirium, cyanosis from severe } \\
\text { congestive heart failure }\end{array}$ & 4 & 4 & 2 & 4 & 4 & 2 \\
\hline Functional, class I & 4 & 4 & 1 & 4 & 4 & 1 \\
\hline Functional, class II and III & 4 & 4 & 2 & 4 & 4 & 3 \\
\hline Functional, class IV & 4 & 4 & 3 & 4 & 4 & 3 \\
\hline
\end{tabular}

From the Canadian Cardiovascular Society, ${ }^{6}$ Raffle (England), ${ }^{7}$ and Doege \& Engelberg (USA). ${ }^{8}$

$1=O K$ to drive without restriction.

2 = Waiting period necessary to observe, investigate, or treat.

$3=$ Disqualified.

$4=$ Not addressed.

*Guidelines from the United States and England provide information only on patients with loss of consciousness. They do not describe steps to take for patients with other conditions listed in this table.

METs less than 2 are so severely impaired that they are considered unable to drive any type of motor vehicle. $^{6}$

In addition to appraisal by exercise testing described above, echocardiographic measurement of left ventricular ejection fraction is also widely available. A measurement of less than $35 \%$, along with appropriate symptoms, can indicate an incapacity to drive a commercial motor vehicle. Despite these laboratory guidelines, however, there are no scientific data correlating a patient's laboratory results with a future risk of a traffic fatality. One must therefore rely chiefly on available expert opinions from several organizations in the form of guidelines and recommendations.

\section{Guidelines for Medical Evaluation}

Guidelines for the medical evaluation of driving capability come from a number of sources. As long ago as 1976 the Medical Commission on Accident Prevention in Great Britain published a review of the medical aspects of fitness to drive. ${ }^{7}$ Ten years later the American Medical Association also published a booklet entitled Medical Conditions Affecting Drivers. ${ }^{8}$ Neither of these important documents has been updated. A 1991 release by the Canadian Medical Association also reviews medical aspects of driving. ${ }^{10}$ It can be downloaded from the Internet at http://www.cma.ca/cpgs/drivexam/index.htm. Each of these reviews addresses the medical evaluation of cardiac patients as well as the evaluation of patients with other medical conditions. More spe- cifically, articles published in 1992 and 1996 from the Canadian Cardiovascular Society, ${ }^{6,11}$ address the medical evaluation of the cardiac patients only and include the recommendations of its consensus conferences. These two articles provide particularly helpful recent information.

All the above guidelines are generally similar and provide a conceptual framework for decision making. Nonetheless, they have not been rigorously tested in clinical practice. A recent report on the clinical practice of specialists suggests tilt-table testing to evaluate simple or vasovagal syncope, although some consider this test to be unnecessary. ${ }^{12}$ In addition to the lack of supporting data on effectiveness of the guidelines, patients could have more than one cardiovascular disorder or other comorbid conditions that adversely impact driving ability. For some conditions, there is divergence of opinion between the guidelines from different countries. For instance, the guidelines from Canada suggest a waiting period of 3 months before driving commercial vehicles after myocardial infarction. ${ }^{6}$ In the US guidelines this waiting period is 12 months. ${ }^{8}$ In England, patients who have had two myocardial infarctions are disqualified from driving a commercial vehicle. ${ }^{7}$

The US guidelines are developed by the American Medical Association and provide only broad suggestions for private vehicle driving, although they contain more detailed recommendations for commercial vehicle driving. ${ }^{8}$ The Canadian guidelines are available from the Canadian Medical As- 
Table 2. Guidelines for Automobile Driving by Patients with Hypertension.

\begin{tabular}{|c|c|c|c|c|c|c|}
\hline \multirow[b]{2}{*}{ Condition } & \multicolumn{3}{|c|}{ Private Vehicle Driving } & \multicolumn{3}{|c|}{ Commercial Vehicle Driving } \\
\hline & USA & England & Canada & USA & England & Canada \\
\hline History of controlled hypertension & 1 & 1 & 1 & 1 & 1 & 1 \\
\hline $\begin{array}{l}\text { Mild hypertension (161-180 } \\
\text { mmHg systolic or } 91-104 \\
\text { mmHg diastolic) }\end{array}$ & 1 & 4 & 1 & 1 & 1 & 1 \\
\hline $\begin{array}{l}\text { Moderate to severe hypertension } \\
\text { (systolic }>180 \mathrm{mmHg} \text { or } \\
\text { diastolic }>104 \mathrm{mmHg} \text { ) }\end{array}$ & 1 & 4 & 1 & 2 & 4 & 2 \\
\hline $\begin{array}{l}\text { Hypertension with vertigo, } \\
\text { faintness, loss of consciousness, } \\
\text { lack of alertness, easy fatigue }\end{array}$ & 2 & 4 & 2 & 2 & 4 & 2 \\
\hline
\end{tabular}

From the Canadian Cardiovascular Society, ${ }^{6}$ Kavanagh et al (England),${ }^{7}$ and Department of ${ }^{\top}$ ransportation ${ }^{1}$ and Doege \& Engelberg (USA). ${ }^{8}$

Note: for private vehicle driving, the guidelines from all three countries recommend driving without restriction for patients with hypertension if they do not have symptoms caused by hypertension or medications. They do not restrict driving based on the level of blood pressure readings. The same is not the case for commercial vehicle driving, wherein certain restrictions apply depending on blood pressure readings.

$1=$ OK to drive without restriction.

$2=$ Waiting period necessary to observe, investigate, or treat.

$3=$ Disqualified.

$4=$ Not addressed.

sociation and from the Canadian Cardiovascular Society, and they are more detailed than the US guidelines. The guidelines for England are issued by the Medical Commission on Accident Prevention and provide detailed suggestions for both the private as well as commercial vehicle driving. ${ }^{7}$ In the United States, many individual states have developed their own guidelines, which can be obtained by contacting the medical board or the medical review section of that state's department of transportation. Despite these limitations, primary care physicians can use the above guidelines for counseling in difficult circumstances until more precise data become available.

\section{Guidelines for Common Conditions}

Most patients with cardiac conditions continue to enjoy safe driving. Certain conditions, however, require a careful evaluation and judgment. Below are presented the recommendations for some of these conditions. The prudent primary care physician should consult these original guidelines in greater detail, particularly when faced with a patient for whom the clinical judgment is difficult. Guidelines for common conditions that might be encountered in primary care settings have been compiled from guidelines from the United
States, ${ }^{1,8}$ England, ${ }^{7}$ and Canada ${ }^{6,10,11}$ and are summarized in Tables 1 through 4.

\section{Hypertension}

Patients who have hypertension are usually asymptomatic and can generally continue to drive safely. For private vehicle driving, there is a uniform opinion that no restrictions are necessary as long as these patients remain symptom-free (Table 2). The requirements for commercial vehicle driving are somewhat more stringent, as shown in Table 2, and a waiting period is often recommended based on the blood pressure readings.

\section{Syncope}

In a study by MacMahon et al, only $40 \%$ of patients reporting syncope were able to drive. ${ }^{13}$ According to the Driver Vehicle Licensing Authority of the United Kingdom, a patient with a syncopal episode of an unknown cause should stop driving for at least 12 months. ${ }^{7}$ For a simple syncopal attack (physiologic or vasovagal), driving need not cease if there is no underlying cardiac disease. ${ }^{7}$ If syncope can be fully controlled by treatment, the patient may drive a private vehicle. ${ }^{10}$ Tilt-table testing is considered unnecessary and impractical in evaluation for fitness to drive. ${ }^{6}$ All the guidelines described above suggest a period of observation for patients with syncope before driving can be resumed (Table 1). 


\begin{tabular}{|c|c|c|c|c|c|c|}
\hline \multirow[b]{2}{*}{ Condition } & \multicolumn{3}{|c|}{ Private Vehicle Driving } & \multicolumn{3}{|c|}{ Commercial Vehicle Driving } \\
\hline & USA & England & Canada & USA & England & Canada \\
\hline $\begin{array}{l}\text { Asymptomatic coronary artery disease } \\
\text { (eg, old myocardial infarction, } \\
\text { abnormal stress test) }\end{array}$ & 1 & 4 & 1 & 4 & 4 & 1 \\
\hline $\begin{array}{l}\text { Coronary artery disease with negative } \\
\text { findings on coronary angiography, } \\
\text { stress radionuclide perfusion, or } \\
\text { stress echocardiography }\end{array}$ & 1 & 4 & 1 & 4 & 4 & 1 \\
\hline $\begin{array}{l}\text { Untreated severe disease of left main } \\
\text { coronary artery }\end{array}$ & 4 & 4 & 2 & 4 & 2 & 2 \\
\hline Coronary artery angioplasty ${ }^{\star}$ & 2 & 4 & 2 & 2 & 2 & 2 \\
\hline Coronary artery bypass surgery ${ }^{\dagger}$ & 2 & 4 & 2 & 2 & 2 & 2 \\
\hline $\begin{array}{l}\text { Myocardial infarction or unstable } \\
\text { angina }^{\ddagger}\end{array}$ & 2 & 2 & 2 & 2 & 4 & 2 \\
\hline
\end{tabular}

From the Canadian Cardiovascular Society, ${ }^{6}$ Raffle (England), ${ }^{7}$ and Doege \& Engelberg (USA). ${ }^{8}$

$1=\mathrm{OK}$ to drive without restriction.

2 = Waiting period necessary to observe, investigate, or treat.

$3=$ Disqualified.

$4=$ Not addressed.

*Canadian guidelines suggest waiting 48 hours before driving private or commercial vehicles. US guidelines suggest waiting 4-6 months before driving commercial vehicles. The UK guidelines suggest waiting 1 year before driving commercial vehicles.

${ }^{\dagger}$ Canadian guidelines suggest waiting 1 month before driving private and commercial vehicles. US and UK guidelines suggest waiting 1 year before driving commercial vehicles.

${ }^{\ddagger}$ Canadian guidelines suggest waiting 1 month before driving private vehicles and 3 months before driving commercial vehicles. US guidelines suggest waiting 12 months before driving commercial vehicles. UK guidelines suggest waiting 2 months for driving private vehicles and disqualify drivers who have had 2 or more myocardial infarctions from driving commercial vehicles.

\section{Coronary Artery Disease}

Because of the likely possibility of ischemia provoked by emotion, driver rage, or the exertion or fatigue of driving; patients with easily provoked angina should stop driving until these symptoms are controlled. ${ }^{7}$ Patients who have stable angina can continue driving if they have periodic medical evaluations. Patients with asymptomatic coronary artery disease or those whose angiography, stress radionuclide perfusion, or stress echocardiographic studies are negative can continue to drive without restrictions based on the available guidelines (Table 3). It should be noted that the guidelines in England disqualify patients who have had two myocardial infarctions from driving a commercial vehicle. A waiting period is generally suggested by the guidelines for patients with untreated severe disease of the left main coronary artery, although the definition of what constitutes severe disease varies somewhat between the guidelines (Table 3). The waiting period varies among different guidelines for patients who have undergone invasive procedures for coronary artery disease and for patients with unstable angina or myocardial infarction (Table 3).

\section{Cardiac Arrbytbmia}

The important consideration for patients with atrial fibrillation, flutter, or reentrant supraventricular tachycardia is whether they have symptoms caused by cerebral hypoperfusion. The incidence of syncope ranges from $33 \%$ to $39 \%$ for patients with atrioventricular nodal reentrant tachycardia, but it is much lower for patients with atrial flutter or atrial fibrillation. ${ }^{14}$ Whether the patient reports episodes of syncope or near-syncope is also of critical importance when evaluating a patient who has a atrioventricular or intraventricular conduction block.

Clearly, patients who are known to have cardiac arrhythmia but are asymptomatic may continue to drive safely, even with known cardiac disease. Most patients with neurally mediated vasovagal or carotid sinus syncope associated with bradyarrhythmia have enough warning before an episode to stop their vehicle. ${ }^{1+}$ Patients with implantable defibrillators usually merit evaluation and clearance by an electrophysiologist. The guidelines for other cardiac arrhythmias and cardiac conduction defects are presented in Table 4. As described in Table 4, different guidelines consistently recommend unre- 
Table 4. Guidelines for Automobile Driving by Patients with Cardiac Arrhythmias.

\begin{tabular}{|c|c|c|c|c|c|c|}
\hline \multirow[b]{2}{*}{ Condition } & \multicolumn{3}{|c|}{ Private Vehicle Driving } & \multicolumn{3}{|c|}{ Commercial Vehicle Driving } \\
\hline & USA & England & Canada & USA & England & Canada \\
\hline $\begin{array}{l}\text { Occasional premature ventricular } \\
\text { or atrial contractions }\end{array}$ & 1 & 4 & 1 & 1 & 1 & 1 \\
\hline $\begin{array}{l}\text { Chronic atrial fibrillation or flutter } \\
\text { without symptoms of underlying } \\
\text { heart disease }\end{array}$ & 4 & 4 & 1 & 4 & 4 & 1 \\
\hline $\begin{array}{l}\text { Sustained supraventricular } \\
\text { tachycardia* }\end{array}$ & 4 & 4 & 2 & 4 & 4 & 2 \\
\hline Sustained ventricular tachycardia ${ }^{\dagger}$ & 4 & 4 & 2 & 4 & 4 & 2 \\
\hline First-degree atrioventricular block & 4 & 4 & 1 & 4 & 4 & 1 \\
\hline $\begin{array}{l}\text { Mobitz type II second-degree } \\
\text { atrioventricular block, } \\
\text { trifascicular block, or a third- } \\
\text { degree atrioventricular block }\end{array}$ & 4 & 2 & 2 & 4 & 4 & 3 \\
\hline Cardiac pacemakers & 2 & 2 & 2 & 4 & 3 & 2 \\
\hline
\end{tabular}

From the Canadian Cardiovascular Society, ${ }^{6}$ Raffle (England), ${ }^{7}$ and Doege \& Engelberg (USA). ${ }^{8}$

$1=\mathrm{OK}$ to drive without restriction.

2 = Waiting period necessary to observe, investigate, or treat.

$3=$ Disqualified.

$4=$ Not addressed.

"Canadian guidelines suggest waiting 3-12 months before driving private vehicles, and a waiting period is necessary for successful treatment with a cardioverter-defibrillator. ${ }^{\dagger}$ Canadian guidelines suggest waiting period for successful treatment with cardioverterdefibrillator before driving private or commercial vehicles.

${ }^{\ddagger}$ Canadian guidelines suggest waiting period for successful treatment with a pacemaker before driving private vehicles.

${ }^{5}$ Canadian guidelines suggest waiting 1 week before driving private vehicles and 3 months before driving commercial vehicles. UK guidelines suggest waiting 1 month before driving private vehicles.

stricted driving for patients with occasional premature ventricular or atrial contractions. Patients with a cardiac pacemaker are disqualified from driving commercial vehicles by the guidelines from England, although the guidelines from the United States and Canada would allow driving if satisfactory functioning of the pacemaker can be established.

\section{Peripberal Vascular Diseases}

Intermittent claudication by itself is not a contraindication to driving. A large aortic aneurysm (>5 $\mathrm{cm}$ ) or one containing a thrombus should probably be repaired before patients can drive safely. ${ }^{10}$ The finding of a small aortic aneurysm, however, does not require revocation of driving privileges. ${ }^{7}$ Deep vein thrombosis can cause embolism with pulmonary infarction, and these patients are advised to cease driving until the condition is successfully treated. ${ }^{10}$ Patients with leg edema or varicose veins may continue to drive unless there is mechanical interference with foot pedal control. ${ }^{10}$

\section{Conclusion}

Primary care physicians will encounter increasingly frequent questions from their patients with cardio- vascular conditions regarding their ability to drive safely. We offer recommendations for driving privilege based upon guidelines from several sources. Primary care physicians can use these guidelines in several ways: to provide reassurance regarding continued safe driving, to require a waiting period of observation before the resumption of driving, to counsel a complete cessation of driving, or to recommend further evaluation by a specialty consultant.

\section{References}

1. Medical advisory criteria for evaluation under 49 CFR Part 391.41. Washington, DC: United States Department of Transportation, Federal Highway Administration, 1998. (Also available at http:// mcregis.fhwa.dot.gov/medical.htm)

2. Drickamer MA, Marottoli RA. Physician responsibility in driver assessment. Am J Med Sci 1993;306: 277-81.

3. Bowen DA. Deaths of drivers of automobiles due to trauma and ischaemic heart disease: a survey and assessment. Forensic Sci 1973;2:285-90.

4. Ostrom $M$, Eriksson A. Natural death while driving. J Forensic Sci 1987;32:988-98.

5. Halinen MO, Jaussi A. Fatal road accidents caused 
by sudden death of the driver in Finland and Vaud, Switzerland. Eur Heart J 1994;15:888-94.

6. Assessment of the cardiac patient for fitness to drive: 1996 update. Can J Cardiol 1996;12:1164-70, $1175-$ 82.

7. Raffle $A B$, editor. Medical aspects of fitness to drive. 3rd edition. London: Medical Commission on Accident Prevention, 1976.

8. Doege TC, Engelberg A, editors. Medical conditions affecting drivers. Chicago: American Medical Association, 1986.

9. Kavanagh T, Matosevic V, Thacker L, Belliard R, Shephard RJ. On-site evaluation of bus drivers with coronary heart disease. J Cardiopulm Rehabil 1998; 18:209-15.
10. Physicians guide to driver examination, 5 th edition. Ottawa: The Canadian Medical Association, 1991. (Available at http://www.cma.ca/cpgs/drivexam/ index.htm)

11. Assessment of the cardiac parient for fitness to drive. Can J Cardiol 1992;8:406-19.

12. Lurie KG, Iskos D, Sakaguchi S, Fahy GJ, Benditt DG. Resumption of motor vehicle operation in vasovagal fainters. Am J Cardiol 1999;83:604-6, A8.

13. MacMahon M, Lawson J, O'Neill D, Kenny RA. Syncope and driving. Age Ageing 1994;23(Suppl 4): S12.

14. Miles WM. Driving issues related to arrhythmic syncope. Cardiol Clin 1997;15:327-39. 